\title{
MODIFIED GOLDEN CODES FOR IMPROVED ERROR RATES THROUGH LOW COMPLEX SPHERE DECODER
}

\author{
K. Thilagam ${ }^{1}$ and K.Jayanthi ${ }^{2}$ \\ ${ }^{1}$ Research Scholar, Department of ECE, \\ Pondicherry Engg. College, Puducherry, India \\ thilagam.kepec.edu \\ ${ }^{2}$ Associate Professor, Department of ECE, \\ Pondicherry Engg. College, Puducherry,India \\ jayanthi@pec.edu
}

\begin{abstract}
In recent years, the golden codes have proven to exhibit a superior performance in a wireless MIMO (Multiple Input Multiple Output) scenario than any other code. However, a serious limitation associated with it is its increased decoding complexity. This paper attempts to resolve this challenge through suitable modification of golden code such that a less complex sphere decoder could be used without much compromising the error rates. In this paper, a minimum polynomial equation is introduced to obtain a reduced golden ratio (RGR) number for golden code which demands only for a low complexity decoding procedure. One of the attractive approaches used in this paper is that the effective channel matrix has been exploited to perform a single symbol wise decoding instead of grouped symbols using a sphere decoder with tree search algorithm. It has been observed that the low decoding complexity of $O\left(q^{1.5}\right)$ is obtained against conventional method of $O\left(q^{2.5}\right)$. Simulation analysis envisages that in addition to reduced decoding, improved error rates is also obtained.
\end{abstract}

\section{KEYWORDS}

Reduced Golden Ratio Golden code (RGR-GC), Bit error rate (BER), effective channel matrix, low complexity decoding, cyclic division algebra (CDA)

\section{INTRODUCTION}

In wireless communication, there is an ever growing demand for higher data rate, highly reliable transmission schemes and increased spectral efficiency for high speed wireless connectivity. Recently, the multiple input multiple output (MIMO) techniques greatly improve the performance as it increases the diversity order of the system. Therefore, the problem of constructing the space time block codes (STBC) with high data rate and good reliability that admits low decoding complexity captures much attention in the literature. For a 2x2 MIMO system, golden code was discussed as a full rate and full diversity code with Non-vanishing minimum determinant (NVD)[1], [2]. In [3], the implementation of simple architecture of a sphere decoder to decode the golden code was introduced. Concatenating the golden code by set partitioning of the inner code and labelling the branches with outer trellis code and its higher

David C. Wyld (Eds) : ICCSEA, SPPR, CSIA, WimoA - 2013

pp. 211-221, 2013. (C) CS \& IT-CSCP 2013

DOI : $10.5121 /$ csit.2013.3522 
trellis complexity was discussed in [4]. In [5], the implementation of the golden ratio encoder such that the exponential accuracy is robust against the threshold effects using Directed acyclic graphs (DAG) model has been discussed. The hardware implementation of Golden code with the Maximum Likelihood [ML] decoding based on sphere decoder was suggested for in [6] and [8]. In [7 ] discusses, the techniques for constructing full-rank, minimal-delay, rate at least one spacetime block codes (STBCs) using commutative division algebras (field extensions) as well as using noncommutative division algebras and also analyzes the coding gain and capacity of these codes. A new ML decoding algorithm for the golden code which retains its fast decodable property on time varying channels and its comparison with the overlaid alamouti code was discussed in [9].The decoding complexity of the golden code was reduced by performing the interleaving process to the co-ordinates prior to encoding to achieve the fast decodability from QAM to arbitrary alphabets was discussed [10]. A decoding technique with the complexity of $\mathrm{O}$ $\left(\mathrm{M}^{2}\right)$ with the trade-off of $2 \mathrm{~dB}$ performance loss using Diophantine approximation was discussed in [11]. The authors in [12]\&[13] discussed, a method to develop the suboptimal decoders for the golden code with reduced complexity and near -ML performance, as the ML- decoding of the golden code have higher complexity. A fast maximum-likelihood decoding algorithm with efficient channel induced by the golden code having a worst-case complexity o $\left(2 \mathrm{M}^{2.5}\right)$ was discussed in [14]. The reduction of average decoding complexity without the performance degradation by an improved sphere decoding for golden code, was discussed in[15]. In [16], the performance analysis of the Golden code in a Bit Interleaved Coded Modulation-MIMO (BICMMIMO) system and its comparison with the uncoded MIMO scheme was discussed. A technique that combines the Golden code with 2x2 multiple beamforming called Golden Coded Multiple Beamforming (GCMB) reduces the decoding complexity compared to general MIMO system using golden code, as discussed in [17]. In [18], the golden code was analysed with application of it to the current scenario of wireless communication. In [19] discusses, the design of low complex Maximum likelihood (ML) decoder for Space Time Block Codes (STBC) and embedded orthogonal STBC with symmetric and asymmetric coding conditions. Multigroup maximum-likelihood (ML) decoding for collocated and distributed space-time block codes (DSTBCs) and the orthogonal frequency-division multiplexing (OFDM)-based Alamouti spacetime coded scheme application in synchronous wireless relay networks also discussed in [20].

The remaining part of the paper is organized as follows: In section 2, the proposed modified golden code is discussed analytically. Section 3, elaborates the decoder design for the proposed setup. Simulation results are discussed in Section 4. Section 5, deals with the conclusion of the paper.

\section{OVERVIEW OF THE CODING SCHEMES}

Over the past few years, it was broadly analyzed that the MIMO techniques greatly improves the system performance and this leads to the choice of better STBC's. There are various types of STBC's namely Alamouti codes, Golden codes, silver code, etc.In order to explain the proposed RGR-GC scheme, a brief introduction to Golden coding method is essential and the details are given in this section.

\subsection{Golden Code}

The golden codes were constructed using a mathematical tool of cyclic division algebras. It uses arbitrary Q-QAM with the base field Q(i), it was built over a quadratic extension, where $\mathrm{i}^{2}=-1$. The key properties of the golden code which improves the performance compared to the other codes [1] are (i) All the codeword have full rank (i.e, non-zero determinant) because of its cyclic division algebraic structure. It transmits two information symbols per channel use (2 $\log 2(\mathrm{Q})$ bits/s/Hz) termed as full rate. Cubic shaping relates to the shape of signal constellation 
and it guarantees no information loss. The minimum determinant remains constant for any QAM size because of its non-vanishing determinant (NVD) property. For 2x2 MIMO systems it achieves the desired diversity multiplexing gain. Mathematically, the golden code with its algebraic properties can be expressed using cyclic division algebra:

$$
\begin{aligned}
& \mathrm{A}=(L / K=Q(i, \sqrt{5}) / Q(i), \sigma, i) \\
& K=Q(i), L=Q(i, \sqrt{5}), \gamma=i \text { and } \gamma \in \mathrm{O}_{K}
\end{aligned}
$$

where, ' $\mathrm{A}$ ' denote the cyclic division algebra, ' $\mathrm{K}$ ' is the algebraic number field, ' $\mathrm{L} / \mathrm{K}$ ' is the field extension, the K-automorphism ' $\sigma$ ' is determined by $\sigma: \sqrt{5} \mapsto-\sqrt{5}$, have the ring of integers

A minimum polynomial equation $\mathrm{X}^{2}-\mathrm{X}-1=0$ is considered and it is solved for the golden ratio.

$$
\theta=\frac{1+\sqrt{5}}{2} \text {, Where, ' } \theta \text { ' is the golden ratio }
$$

A codeword from this algebra, before shaping is of the form

$$
\left[\begin{array}{ll}
a+b \theta & c+d \theta \\
i(c+d \sigma(\theta)) & a+b \sigma(\theta)
\end{array}\right]
$$

Where, the information symbols a,b,c,d €Z[i], ' $\mathrm{i}$ ' is not a norm of any element of $\mathrm{L}$ and $\mathrm{A}$ is the cyclic division algebra. The next section explains the proposed code and its meeting out of the golden code properties with the mathematical expressions.

\subsection{Proposed Reduced Golden Ratio -Golden Code (RGR-GC)}

The Golden code and its codeword format are explained in the aforementioned section. In this section, the proposed code and its properties satisfying golden codes are explained further. The RGR-GC is a scaled version of golden code which is used to normalize the energy and in turn, yields the performance improvement in the system. A reduced golden ratio-golden code (RGRGC) is obtained by choosing a minimum polynomial equation in such a way that, it obtains the reduced golden ratio without losing golden codes key properties. The minimum polynomial equation considered to obtain the reduced golden ratio is $\mathrm{X}^{2}-0.5 \mathrm{x}-0.5=0$.

$\theta=\frac{1+\sqrt{2}}{2}$, Where, ' $\theta$ ' is the reduced golden ratio

Having defined the polynomial and RGR, the next attempt is to focus onto shaping up the process to yield lossless property. A codeword from this algebra, before shaping is of the form

$$
\left[\begin{array}{ll}
a+b \theta & c+d \theta \\
i(c+d \sigma(\theta)) & a+b \sigma(\theta)
\end{array}\right]
$$


where, the information symbols a,b,c,d $€ Z[i]$, ' $\mathrm{i}$ ' is not a norm of any element of $\mathrm{L}$ and $\mathrm{A}$ is the cyclic division algebra.

\section{Property 1: Cubic Shaping}

This section adds cubic shaping to the codebook which provides the information lossless property. For Z[i]-lattice, the codeword is generated by the matrix,

$$
\left(\begin{array}{cc}
1 & \theta \\
1 & \sigma(\theta)
\end{array}\right)
$$

There will be some shaping loss as the matrix is not unitary. These problems can be fixed without losing the other algebraic properties. This issue can be addressed by lattice A with a 'M'generator matrix is given by,

$$
M=\left(\begin{array}{ll}
\alpha & 0 \\
0 & \sigma(\alpha)
\end{array}\right)\left(\begin{array}{ll}
1 & \theta \\
1 & \sigma(\theta)
\end{array}\right)=\left(\begin{array}{ll}
\alpha & \alpha \theta \\
\sigma(\alpha) & \sigma(\alpha) \sigma(\theta)
\end{array}\right)
$$

To make ' $\mathrm{M}$ ' as unitary matrix, the selection is made in such a way that $\alpha \in \mathrm{O}_{L}$

The lattice $\Lambda$ in the determinant form can be written as,

$$
\begin{aligned}
& \operatorname{det}(\Lambda)=\left|N_{L / K}(\alpha)\right|^{2}\left|d_{Q(\sqrt{2})}\right| \\
& =2\left|N_{L / K}(\alpha)\right|^{2}
\end{aligned}
$$

To have the lattice $Z[i]^{2}$, important condition is that the $\operatorname{det}(\Lambda)$ should be a square integer. To find such an element, the factorization of 2 in OL is given by,

$$
2=(1+i-i \theta)^{2}(1-i+i \theta)^{2}
$$

Choosing $(\alpha=1+i-i \theta)$.It can be checked whether the obtained is right lattice using the generator matrix $\mathrm{M}$, where $M^{\dagger}=2 I_{2}$ is obtained by direct computation.

The unitary matrix is $\frac{1}{\sqrt{2}} M$ which yields the shaping property in the first layer and cubic shaping on the other layer. Adding the shaping property, the codeword ' $\mathrm{X}$ ' belonging to the RGR-GC has the form,

$$
X=\frac{1}{\sqrt{2}}\left[\begin{array}{ll}
\alpha(a+b \theta) & \alpha(c+d \theta) \\
i \sigma(\alpha)(c+d \sigma(\theta)) & \sigma(\alpha)(a+b \sigma(\theta))
\end{array}\right]
$$

Where a,b,c,d are QAM symbols and it takes any value in Z[i]. In an infinite code $\mathrm{C}_{\infty}$, a,b,c,d can take any value in $Z[i]$ that is the finite signal constellations are carved from infinite lattices. 


\section{Property 2: Minimum Determinant}

The computation of the minimum determinant of an infinite code is discussed next. Therefore,

$$
X=\frac{1}{\sqrt{2}}\left[\begin{array}{ll}
\alpha & 0 \\
0 & \sigma(\alpha)
\end{array}\right]\left[\begin{array}{lr}
(a+b \theta) & (c+d \theta) \\
i(c+d \sigma(\theta)) & (a+b \sigma(\theta))
\end{array}\right]
$$

Where, $\alpha \sigma(\alpha)=1+i$

$$
\begin{aligned}
& \operatorname{det}(X)=\frac{1+i}{2}[(a+b \theta)(a+b \sigma(\theta))-i(c+d \theta)(c+d \sigma(\theta))] \\
& =\frac{1}{1+i}\left[\left(a^{2}+a b-b^{2}-i\left(c^{2}+c d-d^{2}\right)\right)\right]_{\text {where, }}\left|a^{2}+a b-b^{2}-i\left(c^{2}+c d-d^{2}\right)\right|^{2} \text { is } 1
\end{aligned}
$$

As it is required by the NVD property, the minimum determinant of infinite code is bounded away from zero. To be noted is factor ' $\mathrm{i}$ 'ensures that the average transmitted energy is uniform from both antennas in both channel uses and $|i|^{2}=1$,

Thus,

$$
\delta_{\min }\left(C_{\infty}\right)=\min _{X \neq 0}|\operatorname{det}(X)|^{2}=\frac{1}{2}
$$

The next section explains a new low complexity decoder for the proposed code which is the major task of the golden codes.

\section{PROPOSED LOW COMPLEXITY SPHERE DECODER}

In this section, a new low complexity decoder for the Reduced Golden Ratio- golden codes (RGR-GC) is described. To reduce the decoding complexity, many methods have been analyzed. In [19], the low complexity decoding for the space time block codes and the key properties for reducing the decoding complexity was discussed in broad sense. One of the properties considered in the proposed method is, the real and imaginary parts of the symbol are decoded separately. Moreover, the channel matrix $\mathrm{H}=\mathrm{QR}$, was decomposed using $\mathrm{QR}$ decomposition method, where, ' $Q$ ' is a unitary matrix and ' $R$ ' is an upper triangular matrix. The ' $R$ ' matrix with zero entries and the real elements in it potentially reduce the decoding complexity. In the proposed method, the real and imaginary parts of the symbol are decoded separately using sphere decoder with tree search algorithm, in a single symbol manner for Reduced Golden ratio Golden codes (RGR-GC). The steps involved in the receiver side and algorithm used is explained further. At the receiver, the received vector of samples $y=\left[y_{1}[1], y_{2}[2], y_{3}[3], y_{4}[4]\right]^{T}$ with two antennas at two time instances can be obtained as, $\quad \mathrm{y}=\mathrm{Hx}+\mathrm{n}$

where, ' $\mathrm{x}$ ' is an transmitted symbol vector, ' $\mathrm{n}$ ' is the noise vector and ' $\mathrm{H}$ ' is the effective channel matrix represented as, $X=\left[x_{1}, x_{2}, x_{3}, x_{4}\right]^{T}, n=\left[n_{1}[1], \ldots . n_{2}[2]\right]^{T}$ and

$H=\left[\begin{array}{lcccc}h_{11}[1] & 0 & \phi h_{21}[1] & 0 \\ 0 & h_{21}[2] & 0 & \phi h_{11}[2] \\ h_{12}[1] & 0 & \phi h_{22}[1] & 0 \\ 0 & \phi h_{22}[2] & 0 & \phi h_{12}[2]\end{array}\right]\left[\begin{array}{llll}\alpha & \beta & 0 & 0 \\ -\beta & \alpha & 0 & 0 \\ 0 & 0 & \alpha & \beta \\ 0 & 0 & -\beta & \alpha\end{array}\right]$

respectively. where, $\phi=e^{j \frac{\pi}{4}}, \alpha=\cos (\theta), \beta=\sin (\theta)$ 
In the above equation, $\mathrm{H}=\mathrm{QR}$ is an orthogonal-triangular decomposition of the effective channel matrix where, $\mathrm{Q}$ is unitary and $\mathrm{R}$ is upper triangular. The Gram-Schmidt orthonormalization is followed for the decomposition. Where this procedure is applied to the columns of $\mathrm{H}$, then the entry of $R$ in row $i$ and column $j$ will be the inner product between the $i^{\text {th }}$ column of $Q$ and $j^{\text {th }}$ column of $H$. Therefore, $r_{i, j}=q_{i} * h_{j}$. In direct computation of $R$, the elements $r_{1,2}$ and $r_{3,4}$ are real, through the diagonal elements and the other elements are complex by nature. From the effective channel matrix, the diagonal elements $\left\{r_{1,1} \ldots . . r_{4,4}\right\}$ and nondiagonal elements $r_{1,2}$ and $r_{3,4}$ by direct computation is obtained as,

$$
\begin{aligned}
& r_{11}=\sqrt{\alpha^{2} \gamma_{1}+\beta^{2} \gamma_{2}} \quad, r_{12}=\frac{1}{r_{11} \sqrt{2}}\left(\gamma_{1}-\gamma_{2}\right) \quad r_{22}=\frac{1}{r_{11}} \sqrt{\gamma_{1} \gamma_{2}} \\
& r_{33}=\sqrt{\frac{\alpha^{2}\left(\gamma_{3}+\gamma_{4}\right)}{\gamma_{1}^{2}}+\frac{\beta^{2}\left(\gamma_{5}+\gamma_{6}\right)}{\gamma_{2}^{2}}}, r_{34}=\frac{1}{r_{33} \sqrt{2}} \frac{\left(\gamma_{3}+\gamma_{4}\right)}{\gamma_{1}^{2}}-\frac{\left(\gamma_{5}+\gamma_{6}\right)}{\gamma_{2}{ }^{2}} \\
& r_{44}=\left(\frac{1}{r_{33} \gamma_{1} \gamma_{2}}\right)^{2} \sqrt{\left(\gamma_{3}+\gamma_{4}\right)\left(\gamma_{5}+\gamma_{6}\right)\left(\alpha^{2} \gamma_{2}^{2}\left(\gamma_{3}+\gamma_{4}\right)+\beta^{2} \gamma_{1}^{2}\left(\gamma_{5}+\gamma_{6}\right)\right)}
\end{aligned}
$$

and so on.Decomposing the ' $\mathrm{R}$ ' and ' $\mathrm{X}$ ' matrix, it is got as,

$$
R=\left[\begin{array}{llll}
r_{11} & r_{12} & r_{13} & r_{14} \\
0 & r_{22} & r_{23} & r_{24} \\
0 & 0 & r_{33} & r_{34} \\
0 & 0 & 0 & r_{44}
\end{array}\right], \quad X=\left[\begin{array}{c}
x_{1}^{R}+j x_{1}^{I} \\
x_{2}^{R}+j x_{2}^{I} \\
x_{3}^{R}+j x_{3}^{I} \\
x_{4}^{R}+j x_{4}^{I}
\end{array}\right]
$$

The ' $\mathrm{R}$ ' matrix entries $\mathrm{r}_{11}, \mathrm{r}_{12}, \mathrm{r}_{22}, \mathrm{r}_{33}, \mathrm{r}_{34}, \mathrm{r}_{44}$ are real and in terms of $\mathrm{z}=\mathrm{Q}$ *y, the decoder chooses $\mathrm{x}$ to minimize,

$$
\begin{array}{r}
P(x)=\|y-H x\|^{2} \\
=\|z-R x\|^{2}
\end{array}
$$

where, $\mathrm{P}(\mathrm{x})$ is the noise estimator.

$$
=\left\|z-R_{1} x_{1}-R_{2} x_{2}-R_{3} x_{3}-R_{4} x_{4}\right\|^{2}
$$

The decoder proceeds as follows and the total cost of (14) can be written as,

$$
\begin{aligned}
P(x) & =\left\|v-R_{1} x_{1}\right\|^{2} \\
& =\left\|v^{R}-R_{1} x_{1}^{R}\right\|^{2}+\left\|v^{I}-R_{1} x_{1}^{I}\right\|^{2} \\
v & =z-R_{2} x_{2}-R_{3} x_{3}-R_{4} x_{4}
\end{aligned}
$$

The importance of going from equations (14) to (15) is the fact that $R_{1}$ is real. Therefore, for every candidate $\left(x_{1}, x_{2}, x_{3}, x_{4}\right)$ the fact that $\mathrm{R}_{1}$ is real and allows the receiver to decide on real symbol $\left(x_{1}^{R}\right)$ from the imaginary symbol $\left(x_{1}^{I}\right)$ separately by minimizing $\left|v_{1}^{R}-r_{1,1} x_{1}^{R}\right|^{2}+$ $\left|v_{1}^{I}-r_{1,1} x_{1}^{I}\right|^{2}$. The decoded symbol vector ' $\mathrm{x}$ ' will be the minimum of the overall cost metric $\mathrm{P}(\mathrm{x})$.

The minimum of overall cost metric $\mathrm{P}(\mathrm{x})$ is obtained using the tree search algorithm. A sphere decoder with four level tree, for the reduced golden ratio-golden codes (RGR-GC) associated with different $\mathrm{x}_{\mathrm{i}}(\mathrm{i}=1, . .4)$ at each level has been discussed. It is proposed with a four level tree that associates $d^{R}=x_{4}{ }^{R}$ and $d^{I}=x_{4}{ }^{I}$ in the first level, $c^{R}=x_{3}{ }^{R}$ and $c^{I}=x_{3}{ }^{I}$ in the second level, $b^{R}=$ $\mathrm{x}_{2}{ }^{\mathrm{R}}$ and $\mathrm{b}^{\mathrm{I}}=\mathrm{x}_{2}{ }^{\mathrm{I}}$ in the third level and $\mathrm{a}^{\mathrm{R}}=\mathrm{x}_{1}{ }^{\mathrm{R}}$ and $\mathrm{a}^{\mathrm{I}}=\mathrm{x}_{1}{ }^{\mathrm{I}}$ in the fourth level. Rewriting the cost metric function as, 


$$
\begin{aligned}
& P(X)=\left\|V V^{\prime}-A a^{l}\right\|^{2}+\left\|V^{\prime}-A b^{\prime}\right\|^{2}+\left\|V^{R}-A a^{R}\right\|^{2}+\left\|V^{R}-A b^{R}\right\|^{2}
\end{aligned}
$$

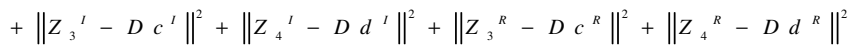

The number of combinations of each symbol $\left(\mathrm{x}_{1}\right)\left(\mathrm{x}_{2}\right)\left(\mathrm{x}_{3}\right)\left(\mathrm{x}_{4}\right)$ is denoted by $q$. The decoding complexity of $\sqrt{q}$, comes from the fact that the symbols $\left(\mathrm{x}_{1}{ }^{\mathrm{R}}\right)$ and $\left(\mathrm{x}_{1}{ }^{\mathrm{I}}\right)$ are separately decodable. Therefore, it has the decoding complexity of $\mathrm{O}\left(q^{1.5}\right)$. The modified golden code and the reduced golden ratio-golden code (RGR-GC) are one and the same is used throughout this paper. For the modified golden codes, a low complex sphere decoder is designed which is the crucial task is discussed further.

\subsection{Tree Search Algorithm}

The figure in Fig.1 represents the tree diagram of the proposed sphere decoder.As explained before, the initial level of tree corresponds to bottom or the starting point of the tree called root node. Each node acts as parent node and the emerging node from the parent node is child node. The final stage of the tree structure is called leaf node. The total cost of a leaf node $\mathrm{x}$ decomposes into the sum of four branch metrics denoted by $\sum P_{i}$. Where, $\mathrm{P}_{\mathrm{i}}$ represents the branch metric for a branch at the (4-i) ${ }^{\text {th }}$ stage of the tree. A sphere decoding algorithm for four level tree structures is briefed. The first few steps involves the initialization (i.e) $Q R$ decomposition of effective channel matrix, computation of ' $\mathrm{Z}$ ' and the sphere radius $\hat{P}$ to infinity. It requires only two sorting operations and incorporates two common optimizations: updating the radius and pruning. The elements $\mathrm{k}, \mathrm{l}, \mathrm{m}, \mathrm{n} € \Pi$ are the permuted channel matrix elements. In the first level of the decoder, the branch metric of the $\mathrm{P}_{4}$ is updated and in turn decides the $\mathrm{x}_{4}{ }^{\mathrm{R}}$ and $\mathrm{x}_{4}{ }^{\mathrm{I}}$. In the second level of the decoder, the branch metric of $\mathrm{P}_{3}$ is updated and $\mathrm{X}_{3}{ }^{\mathrm{R}}, \mathrm{x}_{3}{ }^{\mathrm{I}}$ are estimated. The intermediate values $\mathrm{V}_{2}, \mathrm{~V}_{1}$ are eliminated thereby reducing the interference. In the third level and the fourth level the branch metric $\mathrm{P}_{2}$ and $\mathrm{P}_{1}$ is updated with lowest value and estimates the $\mathrm{x}_{2}{ }^{\mathrm{R}}, \mathrm{x}_{2}{ }^{\mathrm{I}}$ and $\mathrm{x}_{1}{ }^{\mathrm{R}}, \mathrm{x}_{1}{ }^{\mathrm{I}}$ respectively. The overall cost for the current symbol vector is updated. If the current cost $\mathrm{P}$ is less than the previous smallest cost $\mathrm{P}$ then the radius and best candidate vector are updated, otherwise the process is terminated. This procedure is repeated cyclically to decode the symbols.

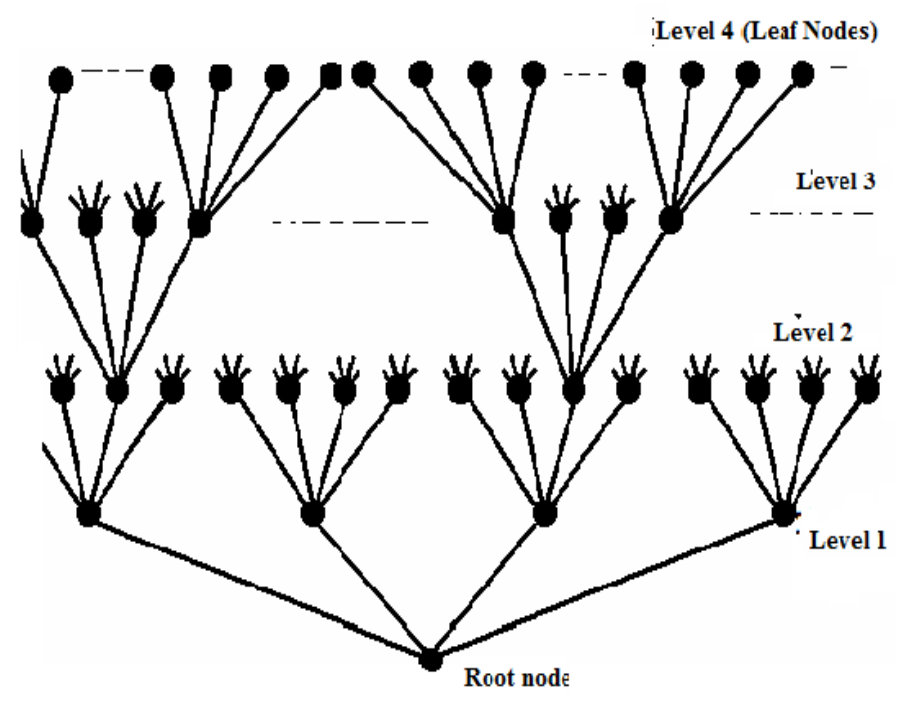

Figure 1. Tree representation of Proposed Sphere decoder 


\section{SIMULATION ANALYSIS}

The simulation analysis for the proposed method is performed with the following parameters and it is tested in MATLAB 7.10 software.

Table 1. Simulation parameters

\begin{tabular}{|l|l|}
\hline \multicolumn{1}{|c|}{ Parameters } & \multicolumn{1}{c|}{ Values/ types } \\
\hline Number of bits & $2 \times 10^{4}$ \\
\hline Number of Transmit antennas & 2 \\
\hline Number of Receive antennas & 2 \\
\hline Modulation schemes & $4-Q A M, Q P S K$ \\
\hline Code rate & $1,1 / 2$ \\
\hline Decoder Types & $\begin{array}{l}\text { Maximum Likelihood, RGR-GC } \\
\text { sphere }\end{array}$ \\
\hline Coding Types & Alamouti , Golden, silver, RGR-GC \\
\hline Channel Type & Rayleigh Fading \\
\hline
\end{tabular}

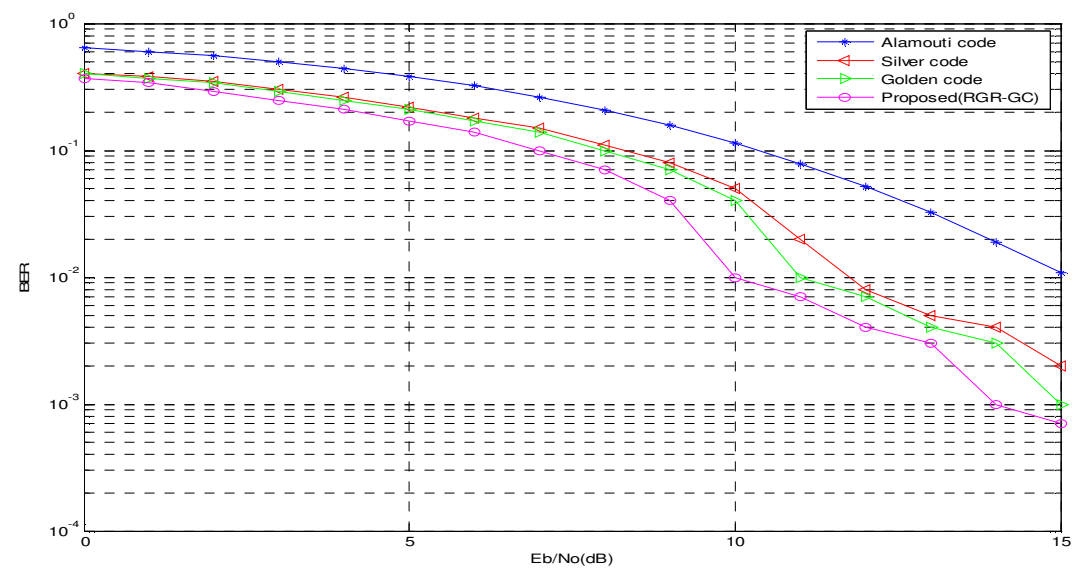

Figure 2. Performance comparison of conventional coding methods with the proposed scheme

From Fig.2.It is inferred that the proposed scheme has better BER performance compared to the conventional STBC coding schemes. The proposed schemes requires $14 \mathrm{~dB}$ (approx) to achieve the BER of $10^{-3}$. The other coding schemes Alamouti, silver and Golden codes requires $15 \mathrm{~dB}$, $16 \mathrm{~dB}$ and $18 \mathrm{~dB}$ (approx) respectively. The proposed scheme uses (RGR-GC) which normalizes the energy and because of that it requires minimum $\mathrm{Eb} / \mathrm{No}$ value compared to the other schemes. 


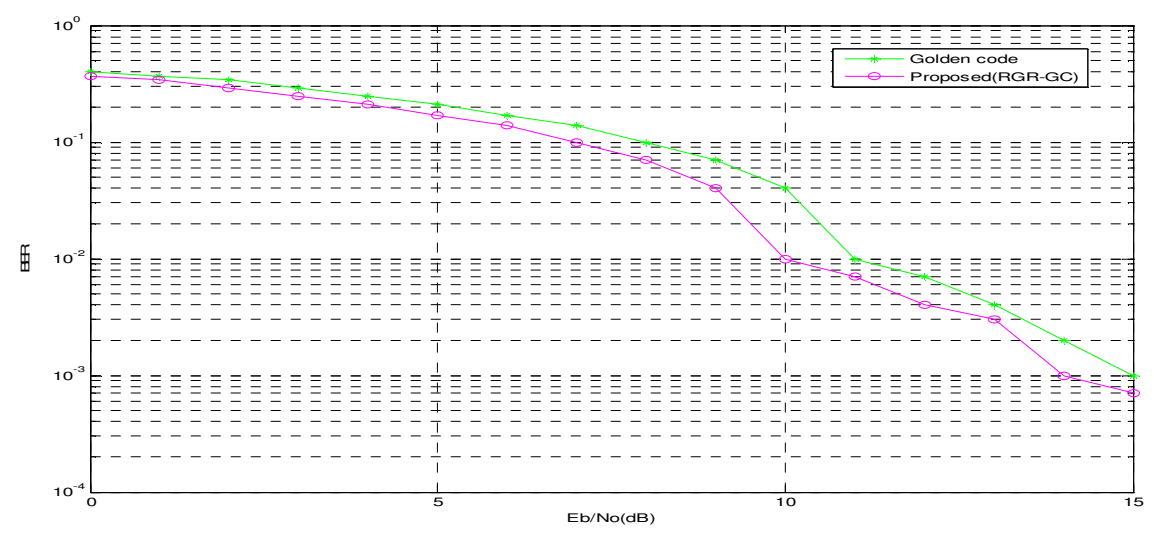

Figure 3. Performance comparison of Golden code with the proposed scheme

From Fig.3. It is inferred that the proposed low complexity sphere decoder is applied to the golden code and the proposed RGR-GC, and the performance graphs were plotted. It is observed that the proposed RGR-GC achieves better BER performance with less decoding complexity compared to the golden code. The proposed logic is also tested for its suitability by separating out the real and imaginary terms of information symbols individually. Although, the data retrieval rate is faster, error rates seems to be very high. Therefore, it is recommended to consider both the real and imaginary terms.

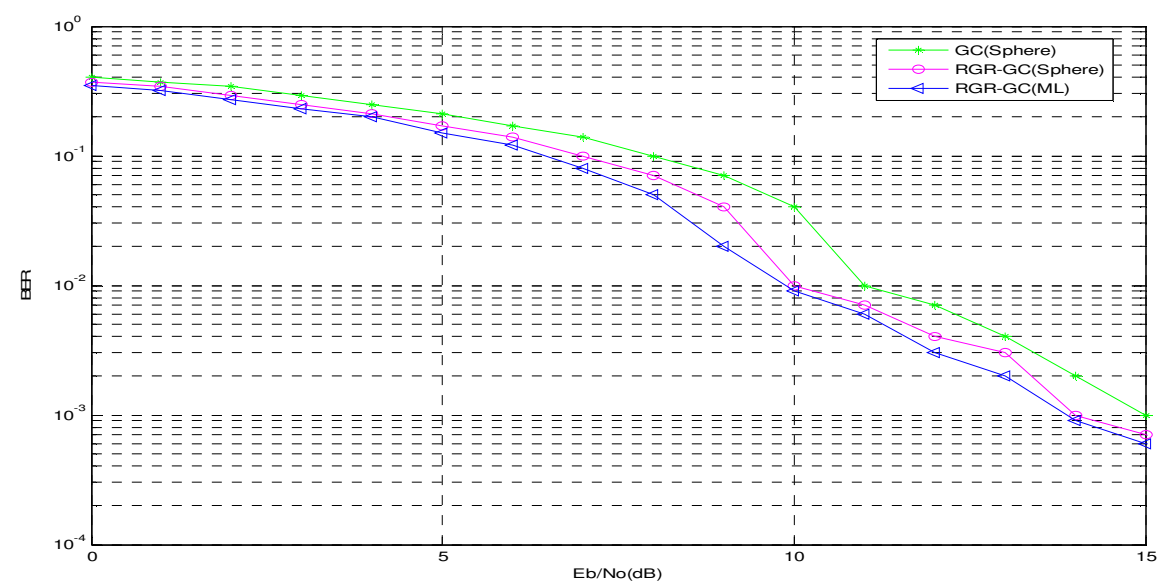

Figure 4. Performance comparison of Proposed (RGR-GC) sphere decoder with the ML decoder

From Fig.4. The performance graphs were plotted for golden codes with proposed low complexity sphere decoder and proposed RGR-GC with conventional ML decoder. It is observed that the proposed RGR-GC with ML decoder achieves better BER performance compared to the other methods. In general ML decoder has better BER performance with less complexity for higher order modulations. So, it is claimed that the proposed RGR-GC sphere decoder achieves near ML performance.

\section{CONCLUSION}

In this paper, a Reduced Golden Ratio-Golden code (RGR-GC) is used with the low complexity sphere decoder which uses tree search algorithm. Introducing RGR-GC yields the scaled version 
of Golden code by a minimum value, which in turn provides an improvement in the BER performance. The proposed decoder separates the complex information symbol into real and imaginary terms and performs decoding in a single symbol wise pattern using the properties of effective channel matrix, so that the decoding complexity is reduced. It is derived analytically that the decoding complexity of the proposed scheme is $\mathrm{O}\left(\mathrm{q}^{1.5}\right)$ where the conventional schemes have $\mathrm{O}\left(\mathrm{q}^{2.5}\right)$. Simulation analysis is done and the simulation results shows that the proposed scheme achieves better BER performance and low decoding complexity, which is the need of the hour for the current MIMO scenario of wireless communication.

\section{REFERENCES}

[1] J.-C. Belfiore, G. Rekaya, and E. Viterbo, "The Golden Code: A $2 \times 2$ full rate Space-Time Code with Non Vanishing Determinants," IEEETrans. on Inf. Theory, vol. 51, no 4, April 2005.

[2] P. Dayal and M. K. Varanasi, "An Optimal Two Transmit Antenna Space-Time Code And Its Stacked Extensions," IEEE Trans. Inf. Theory, vol. 51, n. 12, pp. 4348-4355, Dec. 2005.

[3] B. Cerato, G. Masera, E. Viterbo, "A VLSI Decoder for the Golden code", IEEE Transactions on Information Theory, 2006.

[4] Y.Hong, E.Viterbo and J.C.Belfiore, "Golden Space -Time Trellis Coded Modulation", IEEE Transactions on Information Theory, Vol.56, No. 10, May 2007.

[5] Ingrid Daubechies, C. Sinan Güntürk and Yang Wang, "The Golden Ratio Encoder", IEEE Transactions on Information Theory,Vol. 56, No. 10, October 2010.

[6] J. Jalden and B. Ottersten, "On the complexity of sphere decoding in digital communications," IEEE Trans. Signal Processing, vol. 53, no. 4, pp. 1474-1484, April 2005.

[7] B. A. Sethuraman, B. S. Rajan, and V. Shashidhar, "Full-diversity, highrate space-time block codes from Division algebras," IEEE Trans. Inform. Theory, vol. 49, no. 10, pp. 2596-2616, Oct. 2003.

[8] K. Wong, C. Tsui, R. S. Cheng, and W. Mow, "A VLSI architecture of a K-best lattice decoding algorithm for MIMO channels,” in Proc. IEEE ISCAS'02, 2002, pp. 273-276.

[9] M. O. Sinnokrot and J. R. Barry, "Fast Maximum-Likelihood Decoding of the Golden Code," IEEE Trans. Wireless Commun., vol. 9, no. 1, pp. 26-31, Jan. 2010.

[10] M. O. Sinnokrot and J. R. Barry, "Modified Golden Codes for Fast Decoding on Time-Varying Channels," The 11th International Symposium on Wireless Personal Multimedia Communications (WPMC), Lapland, Finland, September 8-11, 2008.

[11] S. D. Howard, S. Sirianunpiboon, and A. R. Calderbank, "Fast decoding of the golden code by Diophantine approximation," in Proc. IEEE Information Theory Workshop, 2-6 Sept 2007.

[12] M. Sarkiss, J.-C. Belfiore and Y.-W. Yi, "Performance Comparison of Different Golden Code Detectors," in Proc. IEEE PIMRC 2007, Athens,Greece, Sep. 2007, pp. 1-5.

[13] G. R.-B. Othman, L. Luzzi, and J.-C. Belfiore, "Algebraic Reduction for the Golden Code," in Proc. IEEE ICC 2009, Dresden, Germany, Jun.2009, pp. 1-5.

[14] M. O. Sinnokrot and J. R. Barry, "The Golden Code is Fast Decodable," in Proc. IEEE GLOBECOM 2008, New Orleans, LO, USA, Dec. 2008, pp. 1-5.

[15] L. Zhang, B. Li, T. Yuan, X. Zhang, and D. Yang, "Golden Code with Low Complexity Sphere Decoder," in Proc. IEEE PIMRC 2007, Athens, Greece, Sep. 2007, pp. 1-5.

[16] L.Mroueh, S. Rouquette-L'eveil and J.C. Belfiore, "On the performance of the Golden code in BICM-MIMO system and in IEEE 802.11n standard”, IEEE Conference proceedings,Signals,Systems and Computers, 2007, pp. 1544-1548 .

[17] Boyu Li and Ender Ayanoglu, "Golden Coded Multiple Beamforming", IEEE Conference proceedings GLOBECOM 2010.

[18] Emanuele Viterbo, Yi Hong, "Applications of the Golden Code", IEEE Conference proceedings, Information Theory and Applications Workshop,2007,pp.293-300.

[19] Mohanned O. Sinnokrot, "Space-Time Block Codes with Low Maximum-Likelihood Decoding Complexity", Ph.D. Thesis, Georgia Institute of Technology, December 2009.

[20] G.S. Rajan and B.S.Rajan, "Multi-group ML Decodable Collocated and Distributed Space Time Block Codes,” IEEE Trans. on Inf. Theory, vol.56, no.7, pp. 3221-3247, July 2010. 


\section{Authors}

K.Jayanthi received B.E degree from University of Madras in 1997 and M.Tech degree in Electronics and Communication Engineering and Ph.D from Pondicherry University in 1999 and 2007 respectively. She has 13years of experience in teaching / research. She is currently serving as Associate Professor in Department of Electronics and Communication Engineering, Pondicherry Engineering College, Puducherry. To her credit, she has around 12 journal papers and presented 18 papers in International

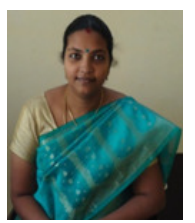
conferences. Her other areas of interest includes satellite communication, Wireless multimedia networks, Spread spectrum communication, Image processing, etc. She has authored a book on Qos Provisioning in Cellular Mobile Networks.

K.Thilagam received her B.Tech and M.Tech degrees in Electronics and Communication Engineering from Pondicherry Engineering College in 2004 and 2007 respectively. She has 5 years of experience in teaching/research. She is currently pursuing Ph.D in the area of Wireless Communication in Department of ECE from Pondicherry Engineering College, Puducherry. Her research activities are focused on Cooperative Communication in Cellular Networks, mainly on Modulation and coding

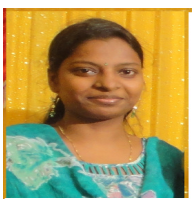
techniques. 